Powerpoint presentation

Useful tips

Conclusion Toolkit is clearly effective as a quality improvement initiative.

Awareness heightened; quality of calls matter for patient safety and professionalism

Strengthened confidence and communication across services

Easily able to be replicated

Easily repeated to ensure improvements are maintained

Could be used as a method of ensuring staff competence as part of Personal Development Plans

Could be used to support mandatory education and training for those services providing 24/7 Telephone Advice

\section{P 172 AN EDUCATIONAL TOOLKIT: SPECIALIST PALLIATIVE CARE TELEPHONE ADVICE LINE}

Chris Haywood, Margaret McConaghy, Anthony Thompson, Clare Molyneux. Willowbrook Hospice, Prescot, UK

\subsection{6/bmjspcare-2014-000654.213}

Background Audit has shown that Integrated Specialist Palliative Care Telephone Advice Lines provide expert support to healthcare professionals caring for patients with palliative and end of life care needs. Governance arrangements including education and support for staff in answering the telephone is variable. A toolkit was devised to support both nursing and medical staff who respond to calls within a Hospice Inpatient Unit.

Aims Produce a toolkit with a clear framework to facilitate, enhance and improve knowledge, skills and confidence of staff in responding to calls made to a Specialist Palliative Care Telephone Advice Line

Aid individual clinical decision making recognising individual limitations; providing guidance on when to escalate to Senior Colleagues

Address individual training needs of staff in a cost effective way

Methods PowerPoint slides for awareness sessions and training

Pre and post evaluation documentation

Combination individual and group training using actual calls

Written reflection with supervisory follow up

Focus on timely, constructive, positive feedback and sharing good practice

Repeated at 6 months

Results Improvements of quality aspect of calls by use of the toolkit

Notable improvements of staff knowledge, skills and confidence Positive impact on Governance

Clearer identification of education and training issues that arise when providing this type of service

The Toolkit Guidance documents

DVD with actual calls

Proforma: reflective practice

Pre $\&$ Post evaluation 